Ann. Génét. Sél. anim., I969, 1 (4), 397-40I.

\title{
VARIATIONS DE L'ÂGE D'APPARITION DU CORNAGE DANS LES RACES CAPRINES ALPINE SAANEN, ALPINE CHAMOISÉE ET POITEVINE
}

\author{
G. RICORDEAU et J. BOUILLON
}

avec la collaboration technique de A. LAJous et P. Guillimin

Station de Génétique quantitative et appliquée, Centre national de Recherches zootechniques, $7 \delta$-Jouy-en-Josas

Institut national de la Recherche agronomique

Station de Testage caprin, 48 - Moissac

\section{RÉSUMÉ}

Chez les Caprins, les cornes ne sont pas toujours visibles à la naissance. Leur âge d'apparition est plus tardif chez les femelles que chez les mâles et varie en fonction inverse du poids de naissance. Le cornage apparaissant au plus tard à 28 jours (dans notre échantillon tout au moins), il faut attendre au moins cet âge pour estimer correctement le nombre de produits cornus dans une population. La présence d'un "épi " de poils à l'emplacement de chaque bourgeon corné peut servir de repère précoce.

\section{INTRODUCTION}

Dans les études sur l'intersexualité associée à l'absence de cornes chez la Chèvre, il est important de connaître sans erreur le cornage des produits pour déterminer correctement le génotype cornage des boucs et les proportions d'animaux cornus dans chaque sexe. Cette note a pour but d'étudier les facteurs de variation de la date d'apparition du cornage et de préciser les précautions à prendre pour enregistrer de façon sûre le cornage des jeunes.

\section{MATÉRIEL ET MÉTHODES}

Les observations portent au total sur 780 produits mâles et femelles appartenant à 3 races et élevés dans deux stations :

- En 1967 et 1968, à la Station de Testage caprin à Moissac (Lozère) sur 396 produits de race alpine Saanen dont 167 cornus;

Annales de Génétique et de Sélection animale. - I969. 
- En 1968 et 1969, au Domaine expérimental de Galle à Avord (Cher) sur 384 produits de race alpine chamoisée et poitevine, dont 246 cornus.

La présence ou l'absence de cornes a été enregistrée à la naissance, puis tous les 3 jours pendant une période d'au moins un mois.

Afin de relier l'âge d'apparition du cornage au phénomène plus général de maturité, on a tenu compte du seul critère disponible, le poids à la naissance.

\section{RÉSULTATS}

Le stade d'apparition du cornage, chez les animaux qui seront cornus, est donné dans le tableau I et les figures I et 2 , en fonction du sexe et du troupeau.

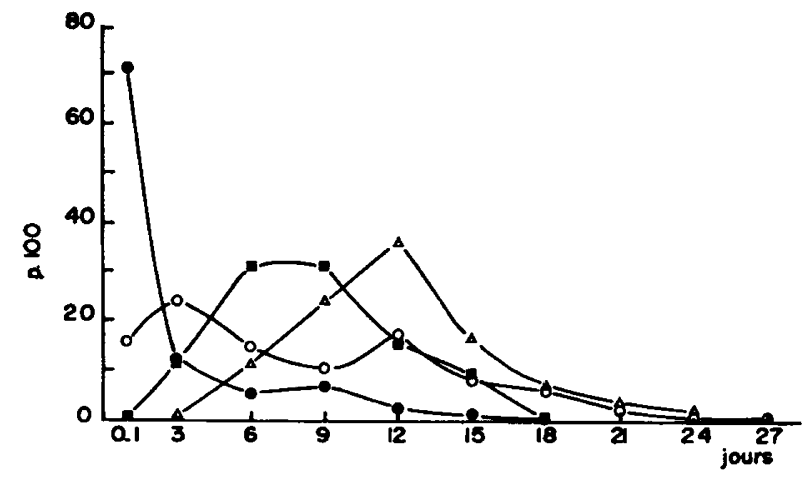

FIG. I. - Age d'apparition $d u$ cornage en fonction du sexe. Fréquence par âge, par sexe et par race.

- ठేర̃ chamoisés et poitevins

- 우 chamoisées et poitevines

- ठ大 saanen

$\triangle$ 우오 saanen.

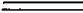

Fig. 2. - Age d'apparition $d u$ cornage : fréquences cumulées par sexe in races Saanen et Chamoisée-Poitevine.

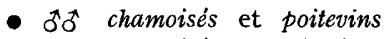

- 우 chamoisées et poitevines

- ठैठ saanen

$\triangle$ 우 saanen

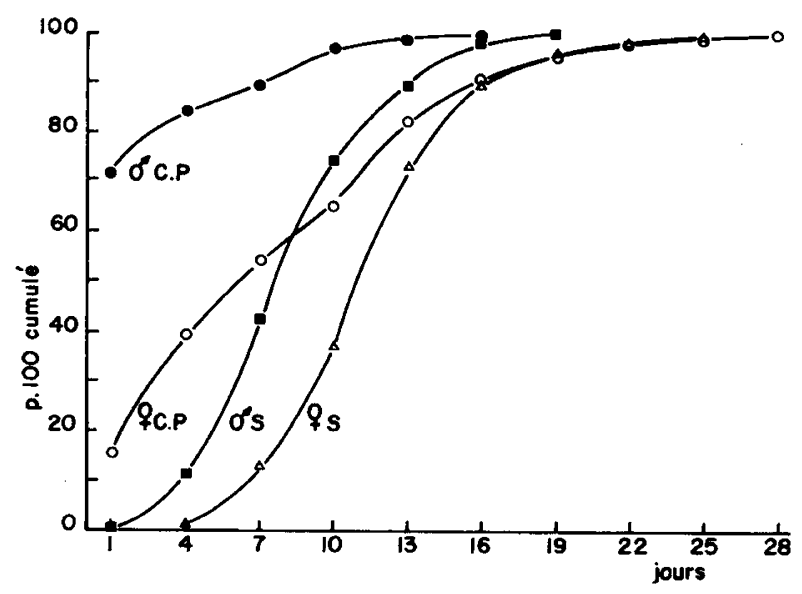


TABLEAU $\mathbf{x}$

Age d'apparition du cornage

\begin{tabular}{|c|c|c|c|c|c|c|}
\hline \multirow{2}{*}{$\begin{array}{l}\text { Age d'apparition } \\
\text { (en jours) }\end{array}$} & \multicolumn{2}{|c|}{$\begin{array}{l}\text { Alpine Saanen } \\
\text { (Moissac) }\end{array}$} & \multicolumn{2}{|c|}{$\begin{array}{c}\text { Alpine chamoisée } \\
\text { (Galle) }\end{array}$} & \multicolumn{2}{|c|}{$\begin{array}{l}\text { Poitevine } \\
\text { [(Galle) }\end{array}$} \\
\hline & $\partial \partial^{*}$ & क̊ & $00^{*}$ & ㅇํ & $\delta \sigma^{\pi}$ & ९̊ \\
\hline $\begin{array}{l}\text { Moyenne } \\
\text { Limites de variation. }\end{array}$ & ${ }_{2} \stackrel{8,5}{a} 18$ & 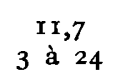 & $\mathrm{o}_{\mathrm{O}}^{2, \mathrm{o}} \mathrm{I}_{4}$ & $\mathrm{o}_{\grave{a}}^{7,4} 28$ & $\begin{array}{l}\mathrm{I,6} \\
\circ \stackrel{a}{I I}\end{array}$ & 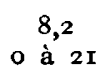 \\
\hline$n \ldots \ldots$ & 89 & $7^{8}$ & 87 & 80 & 46 & 33 \\
\hline
\end{tabular}

La variation du poids à la naissance en fonction de l'âge d'apparition est indiquée dans le tableau 2.

TABLEAU 2

Age d'apparition du cornage et poids à la naissance

\begin{tabular}{|c|c|c|c|c|}
\hline \multirow{2}{*}{$\begin{array}{l}\text { Age d'apparition } \\
\text { (en jours) }\end{array}$} & \multicolumn{2}{|c|}{$\begin{array}{l}\text { Chamoisée } \\
\text { et poitevine } \\
\text { (Galle) }\end{array}$} & \multicolumn{2}{|c|}{$\begin{array}{l}\text { Saanen } \\
\text { (Moissac) }\end{array}$} \\
\hline & $n$ & $\mathrm{~kg}$ & $n$ & $\mathrm{~kg}$ \\
\hline O-I & II 2 & $3,7^{\circ}$ & - & - \\
\hline $2 \mathrm{a} \quad 4$ & 45 & 3,39 & I I & $4, \mathrm{II}$ \\
\hline 5 a 7 & 23 & 3,40 & 36 & 3,54 \\
\hline 8 à Io & $2 \mathrm{I}$ & 3,25 & 45 & 3,38 \\
\hline II d I3 & 22 & 3,36 & 42 & 3,07 \\
\hline 14 à I6 & Io & 2,87 & 21 & 2,93 \\
\hline $\mathrm{I}_{7}$ à $\mathrm{I9}$ & 7 & 2,74 & 6 & 2,60 \\
\hline $20 \mathrm{a} 2 \mathrm{I}$ & 4 & 2,50 & & \\
\hline
\end{tabular}

\section{DISCUSSION}

\section{Influence du sexe}

Aussi bien en race Saanen observée à Moissac, qu'en races chamoisée et poitevine observées à Galle, il existe une différence significative dans l'âge moyen d'apparition du cornage chez les mâles et les femelles. Chez les mâles de ces 3 races, les cornes apparaissent en moyenne 3 à 7 jours plus tôt que chez les femelles.

Dans l'espèce ovine, on constate également des différences entre sexes dans l'expression ou dans la croissance du cornage. Puisque l'ovariectomie n'a pas d'effet apparent sur la formation des cornes et que la castration arrête cette formation, FRASER (r955) pense que les androgènes sont des éléments majeurs de la croissance des cornes. 


\section{Influence de la race}

En race Saanen le cornage n'apparaît que quelques jours après la naissance : à 8 jours en moyenne chez les mâles, à 12 jours chez les femelles, au plus tard 24 jours après la naissance pour ces dernières. En race chamoisée et poitevine, le cornage est souvent présent à la naissance chez les mâles (à 2 jours en moyenne dans les 2 races), mais apparaît plus tardivement chez les femelles : à 7 et 8 jours en moyenne dans les 2 races, au plus tard à l'âge de 28 jours.

Il n'existe donc aucune différence raciale entre chamoisée et poitevine contrôlée dans le même troupeau par les mêmes personnes. La différence entre les Saanen observées à Moissac et les 2 races observées à Galle semble attribuable en majeure partie à l'observateur. En effet, l'appréciation de ce caractère est assez subjective, à la naissance notamment, et certains observateurs ont tendance à anticiper sur l'événement.

\section{Influence du poids à la naissance}

Quel que soit le sexe, le cornage apparaît en moyenne plus tardivement chez les chevreaux de faible poids à la naissance. La régression négative de l'âge d'apparition sur le poids est la même dans les 2 troupeaux. Comme les différences dans le poids de naissance sont dues en grande partie au type de naissance des produits, on peut penser que les chevreaux nés de portées multiples (doubles, triples ou même quadruples) ont une maturité retardée par rapport aux chevreaux nés simples.

\section{Les erreurs commises par défaut dans l'identification du cornage}

En cumulant les données de chaque sexe, dans les 2 populations (fig. 2), il est intéressant de noter que le cornage est visible dans $95 \mathrm{p}$. roo des cas à $\mathrm{I}_{5}$ et I 8 jours pour les mâles et femelles de race Saanen, à 9 et $\mathrm{I} 8$ jours pour les mâles et femelles des races chamoisée et poitevine. Pour être sûr du type de cornage, il faut attendre au moins 4 semaines. Quant aux mâles et aux femelles abattus ou vendus précocement, il est évident qu'on ne peut avoir aucune garantie sur leur type de cornage, sauf s'ils sont déjà cornus. Ces réserves peuvent expliquer pourquoi on trouve souvent un nombre insuffisant de produits cornus par rapport aux effectifs théoriques espérés et une proportion plus élevée de mâles cornus que de femelles cornues (HaUgen, I960; Brandsch, I959, cf. LaUUVERGNe, I969).

\section{Repérage précoce du cornage}

Dove (I935) constate qu'il n'existe pas de véritables " mottes " chez les caprins, puisque même les animaux génétiquement sans cornes présentent 2 protubérances osseuses. Certains éleveurs estiment que si ces bosses sont bien développées et si la peau joue librement sous le doigt, le chevreau sera sans cornes; en revanche, 
si les bosses sont petites et si la peau ne joue pas, le chevreau sera cornu. Cette façon de faire est peu précise et ne peut être valablement retenue.

En r 967 , nous avions observé que la présence d'un épi de poils à l'emplacement de chaque bourgeon corné indiquait presque toujours la pousse ultérieure de cornes. Cette observation a été vérifiée en I968 et I969, puisque sur 595 cas, nous avons enregistré seulement 23 erreurs (soit 3,5\%) dans les 2 sens : I4 jeunes avec épis sur 3 I 8 produits restés mottes et 9 sans épi sur les 277 devenus cornus. La relation entre la présence des épis et le cornage est très bonne, mais l'enregistrement des épis doit être faite soigneusement.

\section{CONCIUSION PRATIQUE}

En enregistrant le cornage à la naissance, ou dans les 48 heures après la naissance comme l'exigent les déclarations de mise-bas, on coit normalement s'attendre à des estimations erronées de la fréquence des cornes. Cependant, la présence d'un épi de poils à l'emplacement de chaque bourgeon corné peut être utilisé comme un repère précoce du phénotype cornu.

Ręu pour publication en octobre 1969.

\section{SUMMARY}

VARIATIONS IN AGE OF HORNING IN BREEDS OF ALPINE SAANEN, ALPINE CHAMOISEE AND POITEVINE GOATS

From observations made during the period between 1967 and 1969, in two herds of 3 breeds of goats (Saanen at Moissac, Chamoisé and Poitevine at Avord), we found on the average that horns appeared earlier in males than in females: average age of 8.5 days in male Saanen goats, 1.8 days in 133 male Chamoisés or Poitevine goats as against 11.7 days (78 subjects) and 7.8 days (113 subjects), respectively, in the females (Table 1 and fig. 1). The differences in breed noticed on one hand in Saanen, and on the other in Chamoisé and Poitevine breeds, seem to be due to the observers, who sometimes anticipated horning. The appearance of horns is probably related to general body development, as is shown in the opposite relationship between age of horning and birth weight (Table 2). Horning became evident at 28 days at the latest (in our sampling), and the goats had to attain at least that age before the number of horned animals in a population could be correctly estimated. However future horned animals may be detected earlier by determination of whorls of hair at the site of the horn buds. In 96.5 per cent of cases (595 observations), these whorls precede the appearance of horns.

\section{RÉFÉRENCES BIBLIOGRAPHIQUES}

BRANDSCH H., I959. Die Vererbung geschlechtlicher Missbildung und des Hornes bei der Hausziege in ihrer gegenseitigen Beziehung. Arch. Geflügelz. Kleintierk., 8, 310-362.

Dove W. I., 1935. Physiology of horn growth. J. exper. Zool., 69, 347-404.

HAUGen E., Ig6o. Hermaphrodisme chez la Chèvre (en norvégien). Meld. Norges LandbrHösk., 39 (ro), I-33.

FraSer A. S., 1955. Growth of horns in Sheep. Austral. J. agric. Res., 6, 770-775.

IAUVERGNE J. J., I969. Fréquence, à l'équilibre, du gène d'origine alpine responsable de l'absence de cornes et de l'intersexualité dans des populations de chèvres. Ann. Génét. Sél. anim., 1, 413-422. 